\title{
Antioxidant activity of wine assessed by different in vitro methods
}

\author{
Chiara Di Lorenzo ${ }^{1}$, Mihaela Badea ${ }^{2}$, Francesca Colombo ${ }^{1}$, Francesca Orgiu ${ }^{1}$, Gianfranco Frigerio ${ }^{1}$, \\ Raul F. Pastor ${ }^{3}$, and Patrizia Restani ${ }^{1}$ \\ ${ }^{1}$ Dipartimento di Scienze Farmacologiche e Biomolecolari, Università degli Studi di Milano, via Balzaretti 9, 20133 Milan, Italy \\ ${ }^{2}$ Faculty of Medicine, Transilvania University of Brasov, Brasov, Romania \\ ${ }^{3}$ Cuarta Cátedra de Medicina Interna, Hospital de Clínicas "José de San Martín”, Universidad de Buenos Aires, Av Córdoba 2351, \\ Buenos Aires, Argentina
}

\begin{abstract}
Epidemiological studies have suggested that a diet rich in antioxidant compounds could help in counteracting the effects of reactive oxygen species, reducing the risk factors for chronic diseases. The moderate consumption of wine, especially red wine, has been associated with the reduction in mortalities from cardiovascular diseases. One of the possible reasons for the protective effect of wine can be identified in the high content of polyphenols (mainly flavonoids), which have significant antioxidant activity. Even though several in vitro tests have been developed for the measure of the antioxidant property, no method has showed a satisfactory correlation with the in vivo situation. On these bases, the aim of this study was the application and comparison of different in vitro methods to assess the antioxidant activity of red, rosé and white wines. The methods were: 1) Folin-Cocalteau's assay for the quantification of total polyphenol content; 2) the DPPH (1,1-diphenyl-2-picrylhydrazyl) spectrophotometric assay and the Trolox Equivalent Antioxidant Capacity (TEAC) spectrophotometric assay for measuring the antioxidant activity of samples; 3) High Performance Thin Layer Chromatography for separation of phenolic substances and assessment of the associated antioxidant activity; 4) electrochemical detection by using a biosensor. Although all the approaches show some limitations, this battery of tests offers a more reliable body of data on the antioxidant activity of vine derivatives.
\end{abstract}

\section{Introduction}

Several epidemiological studies have shown that a nutritionally unbalanced diet contributes to the risk factors for chronic pathological conditions, such as cardiovascular diseases, cancer and diabetes [1].

The production of reactive oxygen species (ROS) is often involved in the initial steps of those conditions, as they can cause the damage of biological structures such as proteins, lipids or DNA. Human metabolism counts on an antioxidant defensive system involving different enzymes to prevent these effects; however, the defences can be overwhelmed in certain conditions (e.g. unhealthy lifestyle and diet) and the progression of oxidative reactions may occur. The inclusion in the diet of foods rich in antioxidant compounds could help in counteracting the toxic effects of ROS. Particularly, over the last decade, the moderate consumption of wine, especially red wine, has been associated with the reduction in mortalities mainly from cardiovascular diseases, slowing LDL oxidation, inhibiting platelet aggregation and stimulating nitric oxide production [2]. Most positive effects of wine are due to the high content of polyphenols (mainly flavonoids), which have shown significant antioxidant properties.

Among them, flavanols [3-O-glycosidesofmyricetin, quercetin,kaempferolandisorhamnetin], flavonols $[(+)-$ catechin, (-)-epicatechin, and (-)-epicatechin gallate] and, in red wines, anthocyanins [3-O-monoglucosidesandthe3-
O-acylatedmonoglucosidesofdelphinidin,cyanidin, petunidin,peonidinandmalvidin] are the main classes of compounds identified and associated with the beneficial effects [3].

On these bases, several studies have been focused on the quantification of antioxidant properties of wines, taking into consideration their polyphenol content. The methods used to assess the antioxidant activity differ from each other in terms of chemical bases and reaction conditions. Since a single assay does not accurately reflect all classes of antioxidant molecules, it is recommended the use of two or more assays in order to better describe the antioxidant property of a specific foodstuff in the human body.

Considering the importance of polyphenols in wine, the aims of this study were: 1) the evaluation of the total phenolic content (TPC) in different wine samples, and 2 ) the set up and application of in vitro methods for a fast screening of antioxidant activity. The methods will include spectrophotometric and chromatographic assays and a novel method, based on the use of an electrochemical biosensor. Biosensors are gaining an increasing role in food analysis; they can be defined as a sub-group of chemical systems, in which the analytical device includes a biological sensor coupled with a chemical or physical transducer. The biosensor used in this study was based on the electrochemical measure of potential to determine concentration of analytes or to characterize the chemical reactivity of a compound. Differential Pulse Voltammetry 
Table 1. Wine samples included in the study.

\begin{tabular}{|l|l|}
\hline Samples analyzed and origin & CODE \\
\hline Red wine Bibeo, Bitonto, Bari & BRe \\
\hline Red wine Spiavento, San Severo, Foggia & SRe \\
\hline Rosé wine Bibeo, Bitonto & BRo \\
\hline Rosé wine Aro, Sassari & ARo \\
\hline White wine Spiavento, San Severo & SW \\
\hline White wine Tuglie, Lecce & TW \\
\hline
\end{tabular}

(DPV) has been used for quantification, since it is suitable to measure the redox properties of chemical compounds having low molecular weights. Applying a potential, a redox reaction occurs on working electrode surface; electrons involved in the reaction modify the current applied in the cell, and this modification is elaborated by a signal transducer.

Results obtained with biosensors were compared with data from spectrophotometric and chromatographic techniques.

\section{Materials and methods}

Fast screening methods of the phenolic fraction of wine samples included: 1) Folin-Ciocalteau's assay for the quantification of total polyphenol content; 2) 1,1diphenyl-2-picrylhydrazyl (DPPH) and Trolox Equivalent Antioxidant Capacity (TEAC) spectrophotometric assays for the assessment of the antioxidant activity of samples; 3) HPTLC (High Performance Thin Layer Chromatography) for the separation and semi-quantitative evaluation of antioxidant property of active compounds. The antioxidant capacity of samples was also evaluated using the electrochemical biosensor described above.

\subsection{Samples}

The wine samples included in the study were from different regions from Southern Italy. The abbreviations used in this study are listed in Table 1.

Samples of wine Bibeo were kindly offered by Francesca Tassiello (Azienda vinicola Pere Rosse, Bitonto, Bari). Other samples were obtained from the market. Before of the analyses, samples were degassed, filtered on a $0.45 \mu \mathrm{m}$ filter (Millipore, Billerica, MA, USA) and maintained at $-20^{\circ} \mathrm{C}$ till the use.

\subsection{Spectrophotometric assays}

Three spectrophotometric assays were used in this study.

\subsubsection{Folin-Ciocalteu's assay}

Total polyphenol content (TPC) was determined according to Singleton and Rossi [4].

Samples were suitable diluted with water for HPLC (VWR, France), and aliquots of $300 \mu \mathrm{L}$ were mixed in test tubes with: $1.5 \mathrm{~mL}$ of Folin-Ciocalteau's reagent (Sigma Aldrich, Germany) diluted 10 times, and $1.2 \mathrm{~mL}$ of $7.5 \%$ sodium carbonate (Sigma Aldrich, Germany). After 30 minutes, the absorbance was measured at $765 \mathrm{~nm}$ in a UV-visible spectrophotometer (Varian Cary 50 SCAN, Palo Alto, California, USA).
Blank was prepared using $300 \mu \mathrm{L}$ of water and treated as described above. Results were expressed as gallic acid (GA) equivalents $(\mathrm{mg} / \mathrm{mL})$.

\subsubsection{Antioxidant activity by DPPH assay}

The antioxidant activity (AOA) of wine samples was evaluated spectrophotometrically, as a measure of radical scavenging activity, using 1,1-diphenyl-2-picryl-hydrazyl free radical (DPPH) $[5,6]$. Aliquots of $1 \mathrm{~mL}$ of DPPH (Sigma Aldrich, Germany) in methanol $(5 \mathrm{mg} / 100 \mathrm{~mL})$ were mixed with $0.5 \mathrm{~mL}$ of each sample suitably diluted. The absorbance was measured after 30 minutes at $517 \mathrm{~nm}$. Results were expressed as $\mathrm{mg} / \mathrm{mL}$ of gallic acid equivalents (GA).

\subsubsection{Antioxidant activity by ABTS assay}

The ABTS assay was performed as described by Re et al. with some modifications [7]. ABTS radical cation solution was prepared by mixing $2.45 \mathrm{mM}$ of potassium persulfate and $7 \mathrm{mM}$ ABTS $(1: 1 \mathrm{v} / \mathrm{v})$; the mixture was then maintained $12-16 \mathrm{~h}$ in the dark at room temperature. Before use, the $\mathrm{ABTS}^{+} \bullet$ solution was diluted with ethanol to reach an absorbance of $0.7 \pm 0.02$ at $734 \mathrm{~nm}$. Aliquots of $1.5 \mathrm{~mL}$ of $\mathrm{ABTS}^{+}{ }^{\bullet}$ solution were mixed with $150 \mu \mathrm{L}$ of each sample suitably diluted. The absorbance was measured after 6 minutes at $734 \mathrm{~nm}$. Results were expressed as $\mathrm{mg} / \mathrm{mL}$ of Trolox equivalents.

\subsection{Fast chromatographic methods: High Performance Thin Layer Chromatography (HPTLC)}

High Performance Thin Layer Chromatography (HPTLC) is a fast and suitable method for the screening of different classes of molecules, allowing the fingerprint characterization of complex products [9]. Furthermore, HPTLC technique can assess some biological properties, which can be directly associated with any specific compound. Among the possible applications, the semiquantitative measure of antioxidant activity is here described.

Particularly, in this study, HPTLC technique was applied to associate antioxidant activity with wine flavonoids separated by chromatography.

\subsubsection{Chromatographic separation of flavonoids and measure of associated antioxidant activity in red, rosé and white wines}

Aliquots of $5 \mu \mathrm{L}$ of standard solutions $(200 \mu \mathrm{g} / \mathrm{mL})$ of the main grape flavonoids (quercetin-3-O-galactoside, quercetin-3-O-glu, quercetin-3-glucuronide, kaemferol3-glu, hyperoside, caftaric acid, epicatechin, epicatechin gallate), and resveratrol were applied on silica-gel plates 254F $(10 \times 20 \mathrm{~cm}$, Merck, Darmstadt, Germany) by using a semi-automatic applicator. For wine samples, volumes of $5 \mu \mathrm{L}$ were loaded onto the plate. The chromatographic run was performed by using a mobile phase $(10 \mathrm{~mL})$ containing acetone:toluene:formic acid 4.5:4.5:1 (v/v/v) [8].

At the end of the chromatographic run, the plate was exposed at $254 \mathrm{~nm}$ (not shown), derivatized with a DPPH (Sigma Aldrich, Germany) methanolic solution (0.05\%) 


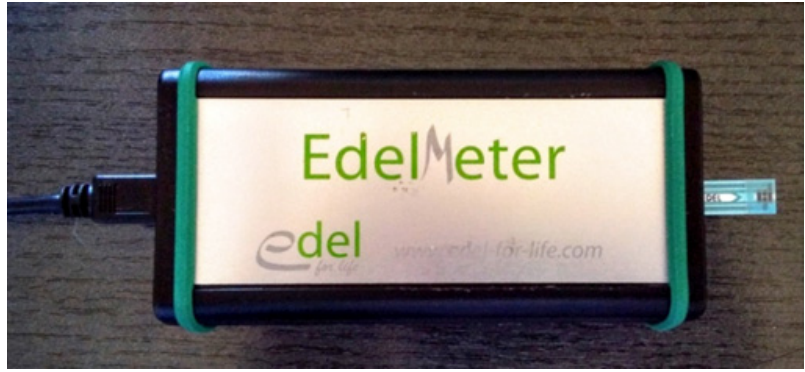

Figure 1. Edel meter with the sensor insert on the right.

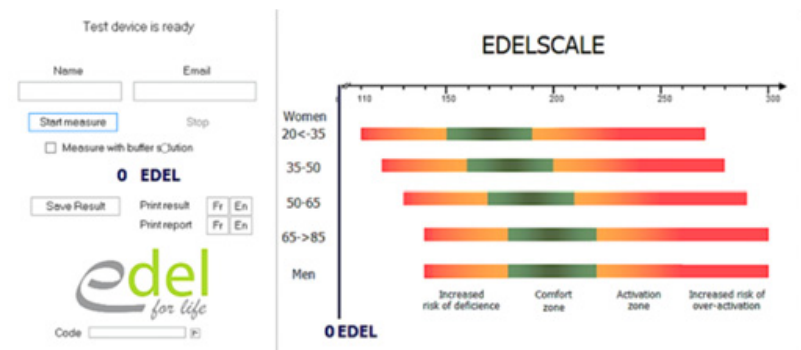

Figure 2. Preview of EDEL program; EDEL values are shown on the left.

and dried for $1 \mathrm{~min}$ at room temperature in an extractor hood. The dried plate was wrapped with aluminium foil for $30 \mathrm{~min}$ and exposed at UV (366 nm) or at visible light. The images were achieved by using a specific software (VisionCats, CAMAG, Muttenz, Switzerland). The band discoloration from violet to yellow was proportional to the antioxidant activity of each phenolic compound.

\subsection{Electrochemical biosensor}

Antioxidant activity of samples was also measured by a new approach based on an electrochemical biosensor, EDEL meter (Edel Therapeutics, Lausanne, Switzerland) an analytical device, which includes a biological detector coupled with a chemical transducer and specific software (Fig. 1).

Gallic acid was used as a reference standard. Working solutions were prepared in phosphate buffer $(\mathrm{pH} 7.4)$. Each sample (standard solutions or diluted wine samples) was transferred in an aluminium-wrapped becker under magnetic stirring. Then, the electrode was inserted in the EDEL meter and immersed in the solutions. After 10 second, the software was launched and measurements, expressed as EDEL units, were recorded each 30s. An example of EDEL scale is reported in Fig. 2. For each sample, the measure was stopped when EDEL values were stable, reaching the plateau status.

The experiments were repeated three times. For each standard concentration, EDEL units were plotted versus time. Then, EDEL values corresponding to the linear part of the curve were used for quantification. The equation $(\mathrm{y}=\mathrm{mx}+\mathrm{q})$ was calculated in order to obtain the slope value $(\mathrm{m})$. At the end of the experiments, a calibration curve was calculated plotting each standard concentration vs the slope values. The calibration line correlated the concentration of standard compounds with slope values. Finally, the same protocol was used for wine samples; the antioxidant activity was measured using gallic acid

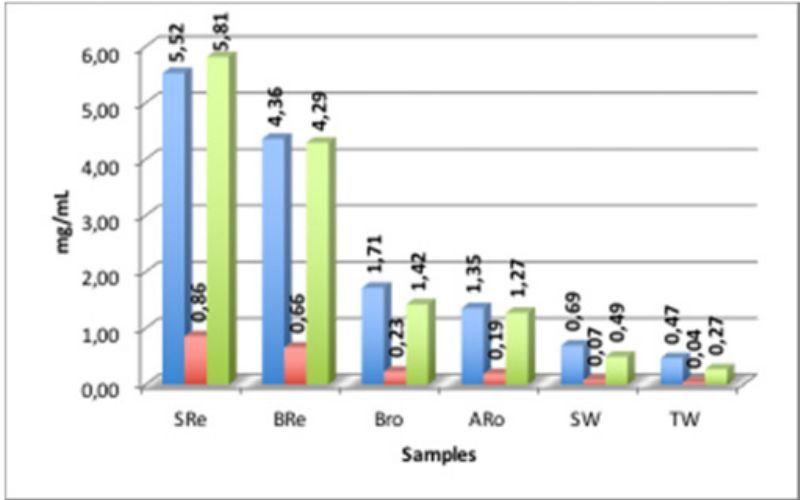

Figure 3. Wine total polyphenol content $(\mathrm{mg} / \mathrm{mL}$ of gallic acid equivalents, blue bars), and antioxidant activity measured with DPPH $(\mathrm{mg} / \mathrm{mL}$ of gallic acid equivalents, red bars) and ABTS (mg/mL of trolox equivalents, green bars) assays. For abbreviations of wine samples see Table 1 .

solutions to prepare the calibration curve. Data were expressed as $\mathrm{mg}$ gallic acid/L.

\section{Results and discussion}

\subsection{Spectrophotometric assays}

Total polyphenol content (TPC) and the antioxidant activity (AOA) quantified using DPPH and ABTS assays are illustrated in Fig. 3.

As expected for the known contribution of anthocyanins, the total phenolic content (TPC) of red wines was higher than that measured in rosé and white wines. The TPC in red wines was eight times and three fold higher than TPC in rosé and white wines, respectively.

The red wines (SRe and BRe) and the rosé wine Bibeo (BRo) showed the highest phenol content: $5.52 \pm$ $0.01,4.36 \pm 0.36$, and $1.71 \pm 0.09 \mathrm{mg} / \mathrm{mL}$, respectively. The lowest polyphenol content was found in white wines Tuglie and Spiavento, and in rosé wine Aro $(0.47 \pm$ $0.03,0.69 \pm 0.02$, and $1.35 \pm 0.10 \mathrm{mg} / \mathrm{mL}$, respectively). These results were in agreement with data published in the scientific literature $[9,10]$.

Although the Folin Ciocalteau's assay lacks of specificity (sugars, ascorbic acid, aromatic amines, and other molecules could interfere), it is a useful test to obtain preliminary information about the total phenolic content of a sample.

In wine samples, antioxidant activity (AOA) measured using DPPH assay ranged between $0.86 \pm 0.03$ (red wine Spiavento) and $0.04 \pm 0.002 \mathrm{mg} / \mathrm{mL}$ (white wine Tuglie), expressed as gallic acid equivalents. The AOA quantified using ABTS assay, and expressed as Trolox equivalents, ranged between $5.81 \pm 0.66$ (red wine Spiavento) and $0.27 \pm 0.02 \mathrm{mg} / \mathrm{mL}$ (white wine Tuglie). Wine samples with the highest content of phenol compounds showed also the highest antioxidant activity. In addition, total phenolic content and the antioxidant activity, measured with both ABTS and DPPH assays, were significantly correlated $\left(\mathrm{R}^{2}>0.99\right)$, as shown in Fig. 4 and Fig. 5.

The high correlation obtained supports the fact that antioxidant activity is mainly due to phenolic compounds.

Differences between DPPH and ABTS results depend on the chemical reagent involved. Both assays are based on 


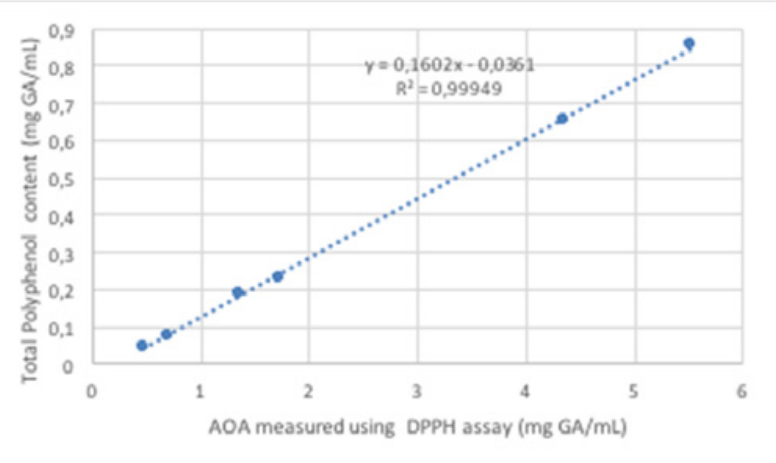

Figure 4. Correlation between total polyphenol content and AOA measured by DPPH assay.

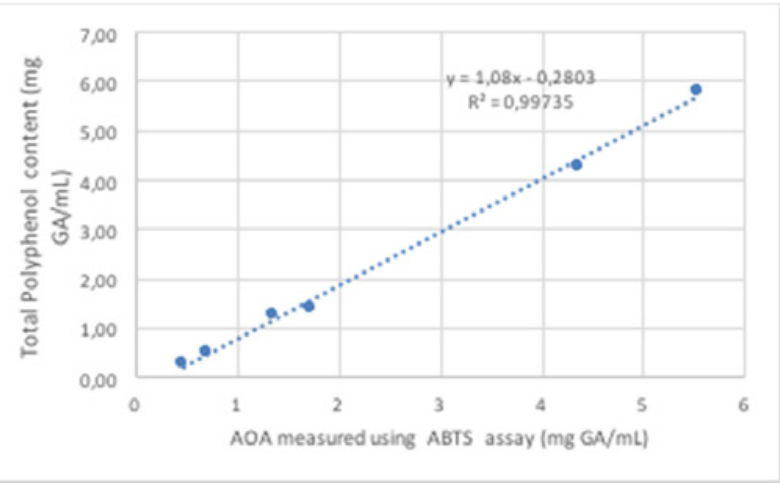

Figure 5. Correlation between total polyphenol content and AOA measured by using ABTS assay.

an electron transfer and involve the reduction of a coloured oxidant. The ABTS assay is based on the generation of a blue/green $\mathrm{ABTS}^{+} \cdot$ that can be reduced by antioxidants, whereas the DPPH assay is based on the reduction of the purple DPPH radical to 1,1-diphenyl-2-picryl hydrazine. Although different, both assays are strongly correlated with TPC and can be considered useful tools in evaluating the antioxidant capacity of foods [11].

\subsection{High performance thin layer chromatography (HPTLC)}

The innovative approach of HPTLC technique allowed the semi-quantitative measure of antioxidant activity associated with a certain number of active compounds.

\subsubsection{Flavonoid pattern and antioxidant activity of wine samples}

HPTLC technique allowed a parallel evaluation of antioxidant activity and flavonoid distribution in wine samples.

In order to perform a semi-quantitative determination of the antioxidant activity, the plate was exposed at visible light after derivatization with the DPPH solution. To obtain a complete evaluation of flavonoid distribution, the plate was also exposed at $366 \mathrm{~nm}$.

Figures 6 and 7 show the flavonoid distribution and the associated antioxidant activity of the wine samples included in this study.

After derivatization with DPPH solution and exposure of the plate at $366 \mathrm{~nm}$ (Fig. 7), a band corresponding to

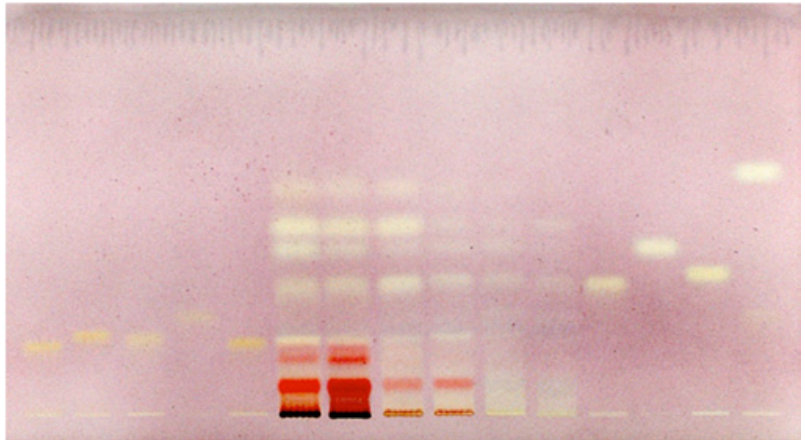

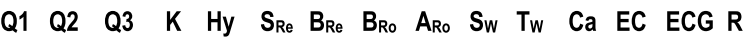

Figure 6. HPTLC patterns of wine samples at visible light after derivatization with DPPH solution. Standard flavonoids are run in parallel.

$$
\begin{array}{ll}
\text { Q1 = Quercetin-3-O-galactoside } & \text { Aro = Rosé - Aro } \\
\text { Q2 = Quercetin-3-O-glucoside } & \text { SW }=\text { White - Spiavento } \\
\text { Q3 = Quercetin-3-O-glucuronide } & \text { TW }=\text { White - Tuglie } \\
\text { K = Kaempferol-3-O-glucoside } & \text { Ca = Caftaric acid } \\
\text { Hy = Hyperoside } & \text { EC = Epicatechin } \\
\text { Sre = Red - Spiavento } & \text { ECG = Epicatecingallate } \\
\text { BRe = Red - Bibeo } & \text { R = Resveratrol } \\
\text { BRo = Rosé - Bibeo } &
\end{array}
$$

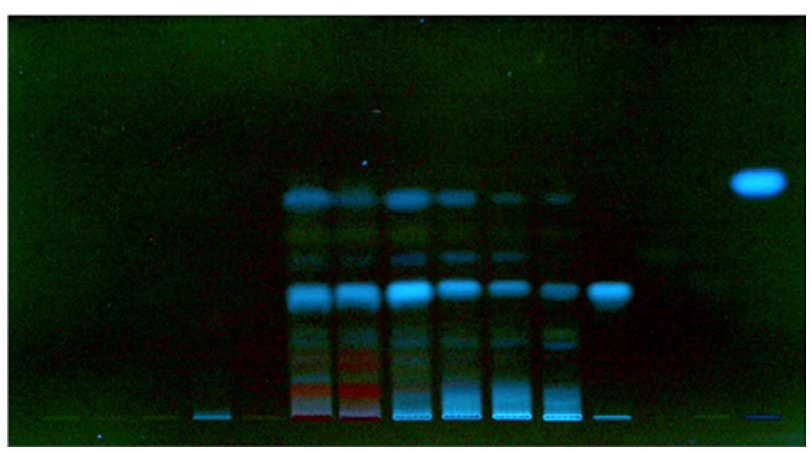

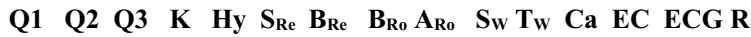

Figure 7. HPTLC patterns of wine samples at $366 \mathrm{~nm}$ after derivatization with DPPH solution. Standard flavonoids are run in parallel. For abbreviation see Fig. 6.

caftaric acid was visible in all samples; this compound was the most abundant and characteristic phenol compound in Rosé wine Bibeo and red wines (SRe and BRe), where the highest antioxidant activity was also observed (Fig. 6). At visible light, hyperoside and epicatechin are clearly detectable in red and rosé wines, both showing also high antioxidant activity. White wines showed the lowest level of flavonoids.

Table 2 shows the relative abundance of antioxidant compounds in wine samples evaluated by HPTLC, in comparison with the quantitative determination of total antioxidant activity measured by spectrophotometric methods.

\subsection{Electrochemical biosensor}

Figure 8 shows the trend of Edel units versus time, for each gallic acid concentration used.

The preliminary data obtained with this method showed a high variability in the antioxidant activity of samples, in particular for the red wines SRe and BRe: 
Table 2. Comparison between the different analytical approaches for the antioxidant activity evaluation.

\begin{tabular}{|c|c|c|c|}
\hline Samples (Code) & HPTLC (relative abundance) & DPPH assay (mg gallic acid /mL) & ABTS assay (mg trolox /mL) \\
\hline SRe & ++++ & 0.86 & 5.81 \\
\hline BRe & ++++ & 0.66 & 4.29 \\
\hline BRo & +++ & 0.28 & 1.42 \\
\hline Aro & ++ & 0.19 & 1.27 \\
\hline SW & + & 0.07 & 0.49 \\
\hline TW & + & 0.04 & 0.27 \\
\hline
\end{tabular}

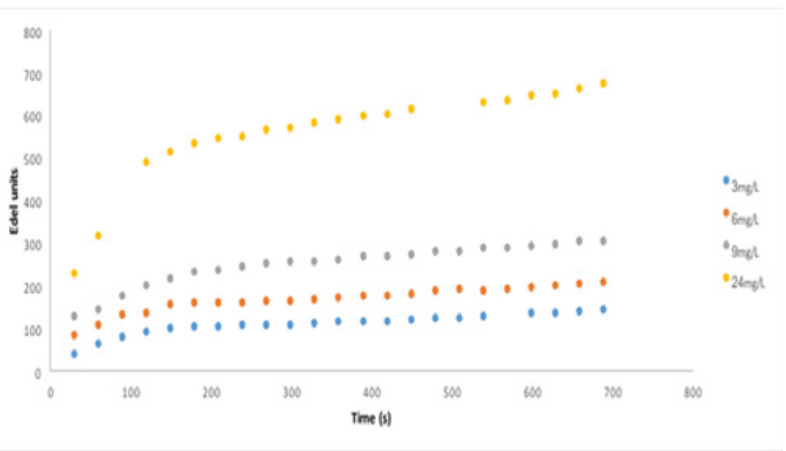

Figure 8. Increase of Edel units for different gallic acid concentrations in function of time.

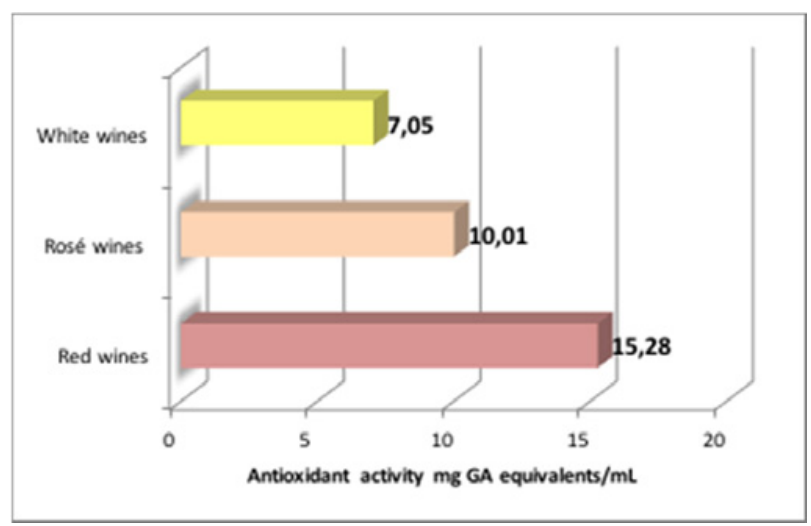

Figure 9. Mean antioxidant activity in the three classes of wines.

22.54 and $8.01 \mathrm{mg}$ gallic acid equivalents $/ \mathrm{mL}$, respectively. On the other hand, results for white $(5.34$ and $8.76 \mathrm{mg}$ $\mathrm{GA} / \mathrm{mL}$ ) and rosé wines $(10.26$ and $9.76 \mathrm{mg} \mathrm{GA} / \mathrm{mL}$ ) were well correlated with data obtained by spectrophotometric assays. Since biosensors have being only recently applied to the measure of antioxidant activity, no literature reference was at disposal for comparison.

However, considering the mean AOA values for the different types of wine (red, rosé and white) a good correlation can be found with DPPH and ABTS analysis (Fig. 9).

It should be noticed that this method is still in development and a higher number of samples is required to validate the results obtained.

\section{Conclusions}

In the present study, different analytical approaches have been applied to assess the antioxidant activity in wine.
The Folin-Ciocalteu's method allowed to obtain a fast quantification of total polyphenol content and the data produced showed to be highly correlated with antioxidant activity measured by two different spectrophotometric assays. As expected, red wines showed the highest antioxidant activity, followed by rosé and white wines.

Although not specific, spectrophotometric methods were useful to obtain preliminary information on the phenolic composition of wines and the associated antioxidant activity. The HPTLC showed interesting results not only for the screening of active compounds, but also for the evaluation of their specific antioxidant activity; the most usual in vitro assays determine only the total activity.

Notably, rosé wines (mainly BRo) showed an interesting profile for both the total polyphenol content and the antioxidant activity, mainly due to the presence of caftaric acid and epicatechin.

A new analytical approach, based on the use of an electrochemical biosensor was also used. Although with some limitations, probably due to the complexity of the matrix and to possible interferences with the biological nature of sensor, preliminary data have shown that it could be a promising tool to assess antioxidant activity of wines. In addition, if compared with other methods, it does not require specific reagents and the portability makes this device suitable for controls performed in wine cellars.

All analytical methods presented in this study showed a similar and comparable trend, so that they could offer a reliable body of data in the evaluation of antioxidant activity in vine derivatives.

\section{References}

[1] M.S. Fernàndez-Pachòn, D. Villano, M.C. GarcìaParilla, A. M. Troncoso, Anal. Chim. Acta 513, 113 (2004)

[2] G.J. Soleas, G. Tomlison, E.P. Diamandis, D.M. Goldberg, J. Agric. Food Chem. 45, 3995 (1997)

[3] C. Stockley, P.L. Teissedre, M. Boban, C. Di Lorenzo, P. Restani, Food Funct. 3, 995 (2012)

[4] V. L. Singleton, J.A. Rossi, Am. J. Enol. Viticult. 16, 144 (1965)

[5] W. Brand-Williams, M.E. Cuvelier, C. Berset, Food Sci. Technol. 28, 25 (1995)

[6] L.P. Leong, G. Shui. Food Chem. 76, 69 (2002)

[7] R. Re, N. Pellegrini, A. proteggente, A. Pannala, M. Yang, C. Rice-Evans, Free Radical Biol. Med. 26, 1231 (1999)

[8] E. Reich, A. Schibli, 2006. High Performance ThinLayer Chromatography for the Analysis of Medicinal Plants (CIC Edizione internazionale, 2006) 
[9] E. Büyüktuncel, E. Porgal1, C. Çolak, Food and Nutrition Sciences 5, 1660-1667 (2014)

[10] M.S. Fernández-Pachón, D. Villaño, M.C. GarcìaParrilla, A.M. Troncoso, Analytica Chimica Acta 513, 113-118 (2004)
[11] A. Floegel, D. Kim, S. Chung, S. I. Koo, O. K. Chun, Journal of Food Composition and Analysis 24, 1043-1048 (2011) 